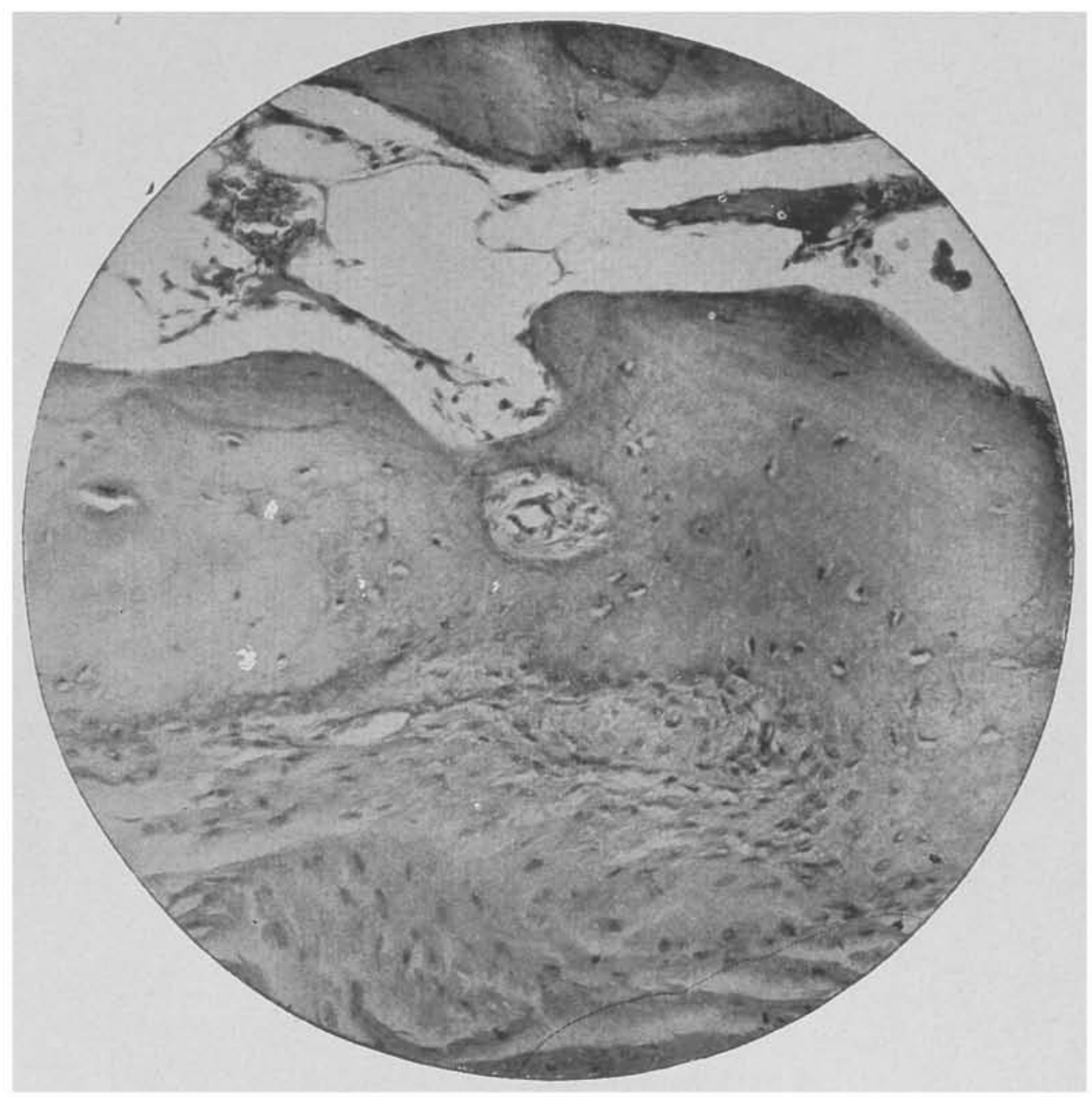

S. $1113-24$.

Augmento: 80 diam. 



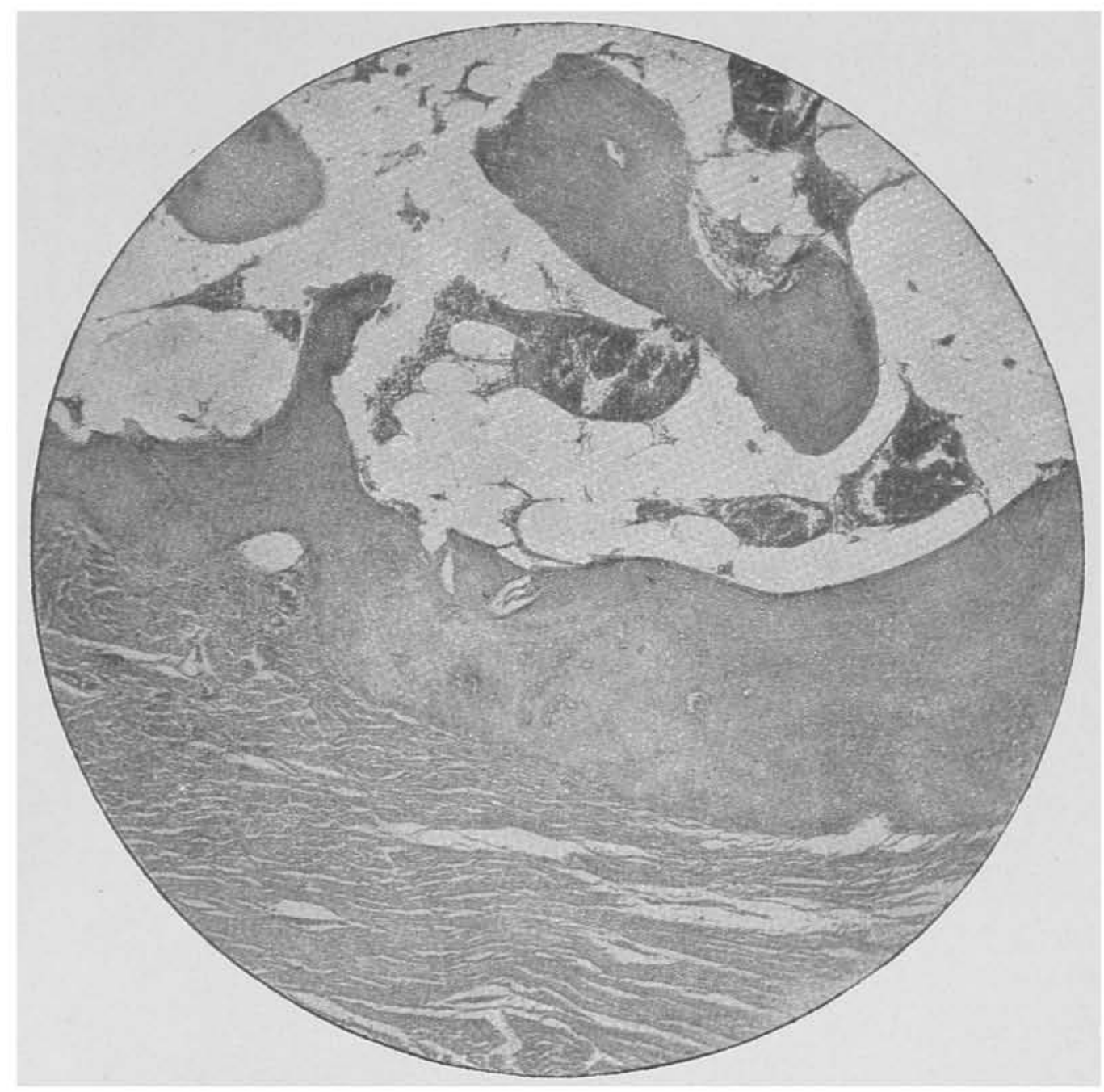

S. $1113-24$.

Augmento: 210 diam. 



\section{SOBRE UM CASO DE MYOSITE OSSIFICANTE}

\section{Trabalho do Instituto Anatomo-Pathologico da Faculdade de Medicina e Cirurgia de São Paulo.}

Exporemos succintamente um caso por nós observado no serviço do Prof. B. Montenegro e em seguida faremos algumas considerações relativas ao modo de formação e á signifiçação desses nódulos osseos.

Anamnese: Sr. T. L. C. Brasileiro, com 42 annos de idade, solteiro. Dos seus antecedentes hereditarios nada ha que nos posía interessar em relação ao caso presente.

Quanto aos antecedentes pessoae ${ }_{\tilde{s}}$ conta-nos ter tido varias molestias proprias da infancia. Mais tarde foi victima do impaludismo, febre amarella, typho e por fim contrahiu a syphilis.

Apoz essa infecção syphilitica e máu grado o. tratamento começou a sentir dore s rheumatoides generalisadas. No joelho esquerdo as dores eram mais fortes e acompanhadas de sensação de fraqueza.

Em consequencia desse incommodo soffreu o nosso doente uma queda de modo a cahir com o peso do corpo sobre o calcanhar ésquerdo, estando com a perna em hyper-flexão sobre a coxa, produzindo-se então uma ruptura do tendão quadricipital interessando fibras musculares.

Tratado convenientemente esse incidente, notou, um mez e meio depois, o apparecimento de um nodulo duro, dez centimetros acima da rotula, nodu!o esse que começou a cres̃cer progressivamente.

Da sua situação a radiographia nos dá amplos esclarecimentos, assim como, juntamente com os dados anamnesicos, nos conduz ao diagnostico de myosite osíificante circumscripta.

Como esses nodulos produzisisem disturbios funccionaes aconselhou o Prof. B. Montenegro a intervenção cirurgica que se realisou, por nćs auxiliada, no Sanatorio Santa Catharisa.

A operação consistiu na extirpação dos noduloß osseos juntamente com a porção de tendão quadriciptal na qual residia o processo.

Pelo exame anatomo-pathologico a que procedemos encontramos cuas placas duras medindo $3,5 \mathrm{cms}$. por $2 \mathrm{cms}$. e $2 \mathrm{cms}$. por $1 \mathrm{~cm}$. situadas na espessura do tendão, proximo á inserção muscular. 
Um corte macroscopico mostra-nos a estructura de um osso es;onjoso com medulla joven.

Pelo exame microscopico pudemos observar a presença de trabeculas osgeas em via de formação por processo de metaplasia ossea conjunctiva. Mui nitidamente se viam as phases successivas dessa osteogenese activa e progressiva.

Os pequenos vasos circumvisinhos aos nodulos osseos mostravam um manguito parvicellular de peri-vascularite chronica.

Para explicar a origem dessas formações foram aventadas tres theorias: $1 .^{\circ}$ ) Ossificação de unı hematoma. $2 .^{\circ}$ ) Semeadura periostica. $3 .^{\circ}$ ) Myosite ossificante.

A theoria da.ossificação de um hematoma, erronea, não é mais acceita.

A segunda theoria ou da semeadura periostica faz o nodulo ter por matriz pequenos fragmentos de periosteo levados ao seio da massa muscular.

Essa theoria explica os nodulos osseos dos adductores, e mesmo os nodulos intra-musculares diz Orlow provirem do periosteo arrancado e levado para a espessura do musculo, pela retracção das fibras tractoras.

No sentido de confirmar essa noção Berthier e Siens rea'isaram experiencias, nas quaes conseguiram obter producções osiseas pela semeadura experimental de fragmentos de periosteo joven ein pleno musculo.

Essa explicação é inapplicavel, porém, aos casos dos nodulos tendinosos, como a nosso, e ainda quando sendo o nodulo intramuscular é o musculo separado do osso por tendão.

Emfim, temos ainda a objeç̧ão que o nódulos de Berthier e Siens é reabsorvido posteriormente.

Por ultimo resta-nos analysar a theoria da myosite ossificante que admitte a interferencia de uma inflammação com o caracter especial de produzir tecido osseo.

Essa theoria é assim vagamente exposta pelos varios auctores, sem que nenhum delles entre na analyse do mecanismo intimo da formação do tecido osseo.

A exposição de idéas nosisas sobre esse assumpto é o principal, senão unico, motivo da publicação deste artigo.

Admittimos que sob a influencia de um traumatismo unico ou de traumatismos repetidos, ou ainda em consequencia de uma ruptura, produz-se um eastado inflammatorio local que se caracterisa, como as demais inflammações, por phenomenos alterativos, exsudativos e proliferativos. 


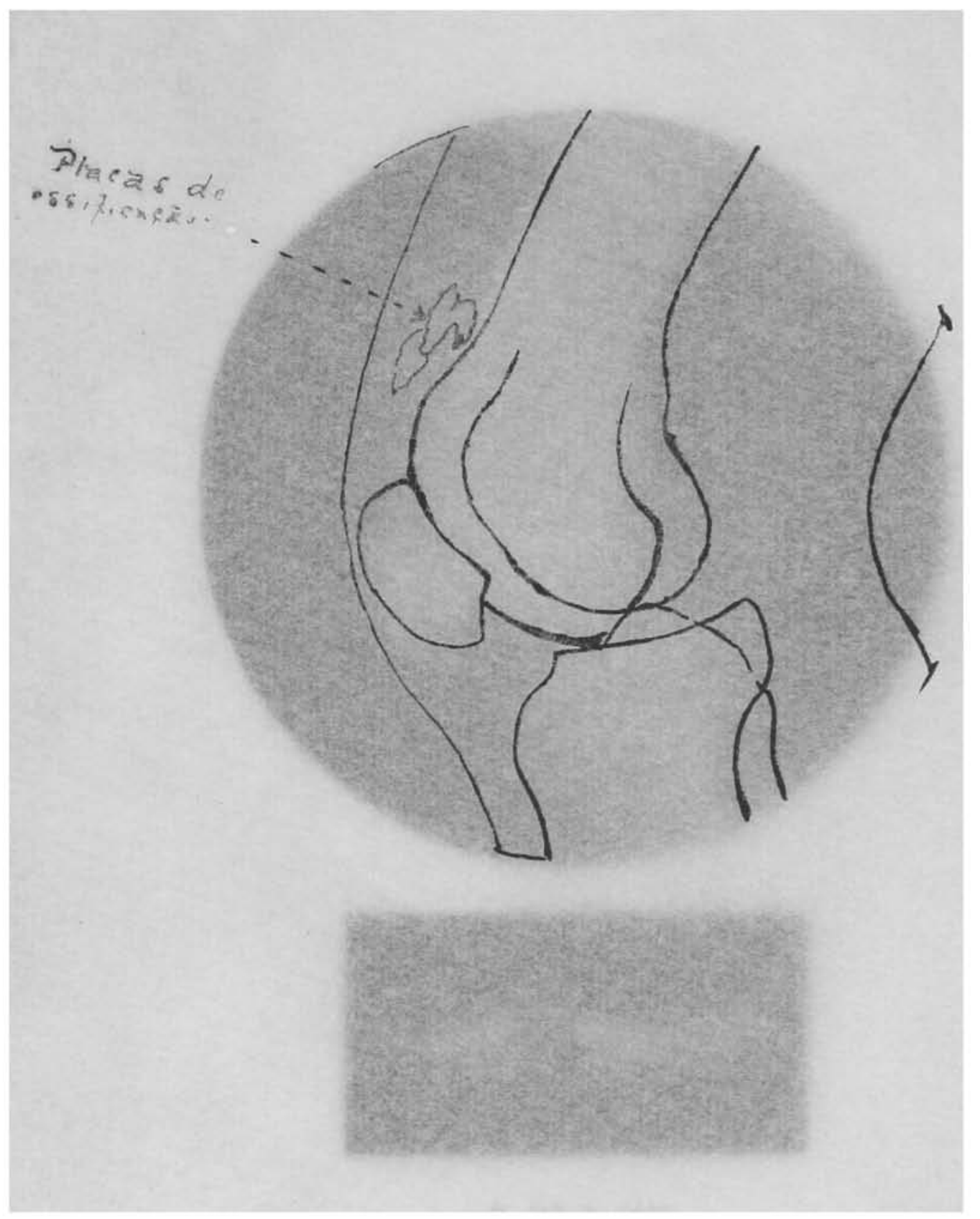


12

Revista de Medtetra

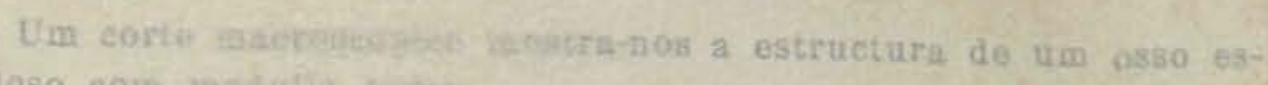

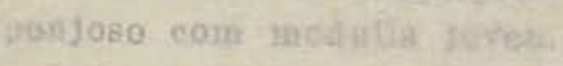

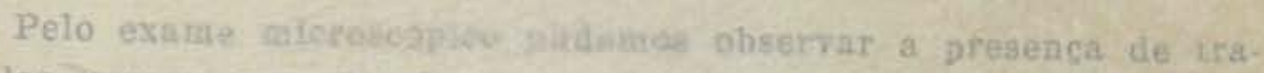

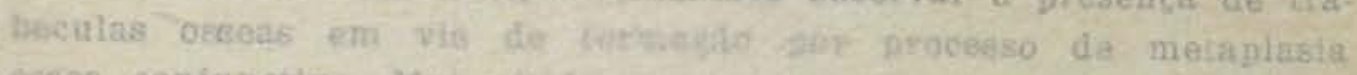

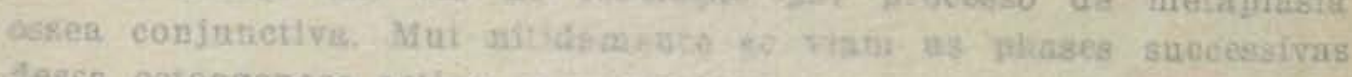
Lessa osteogenese activa et prosrostre

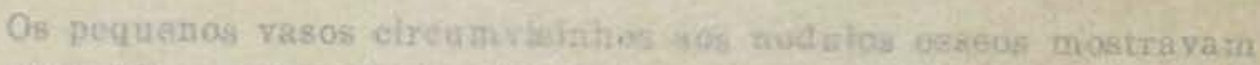

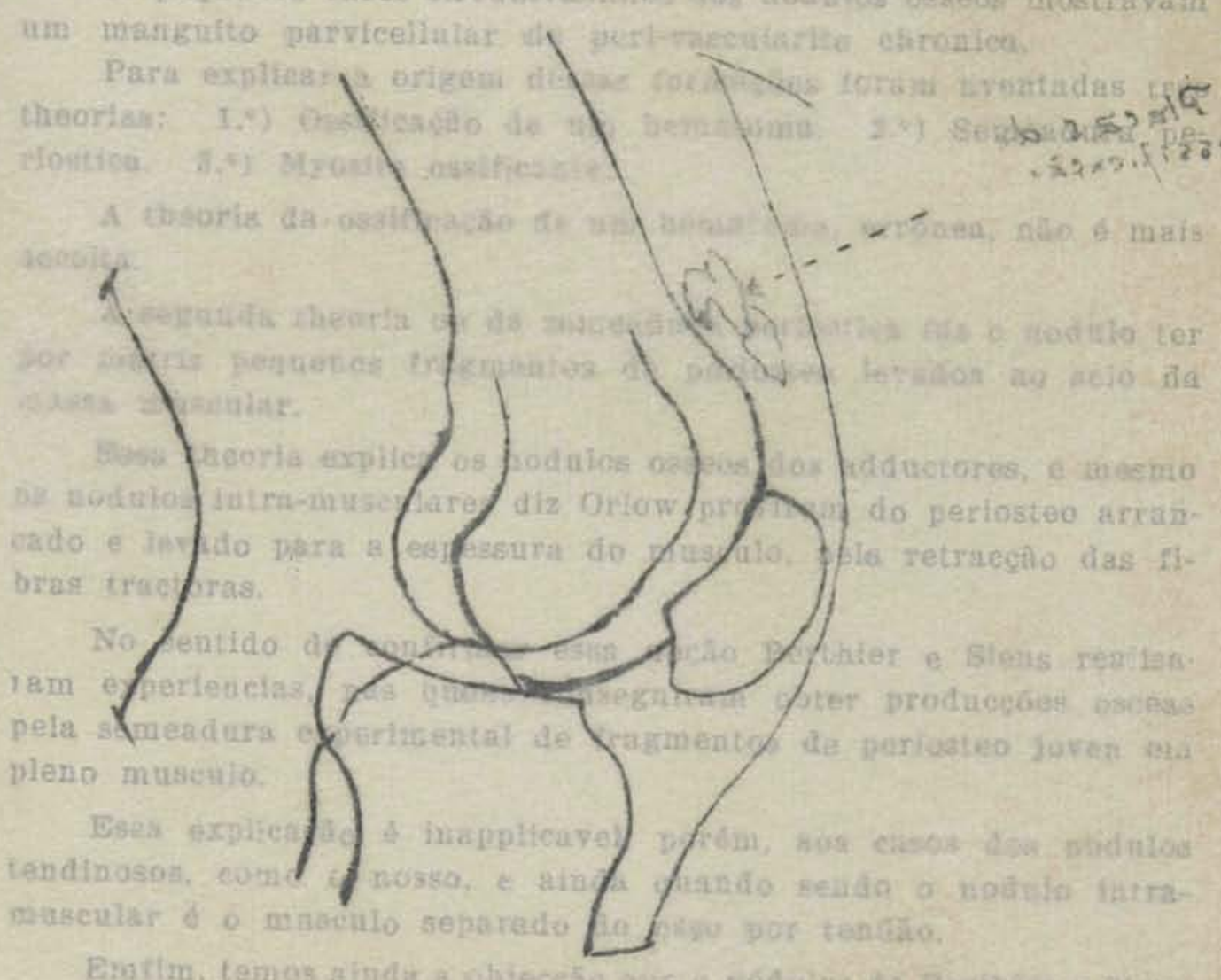

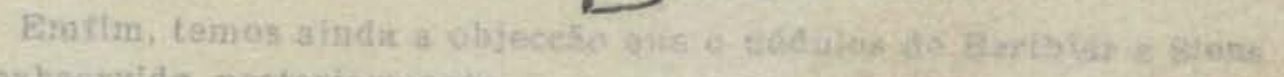
reabisorvido pasterionimezite.

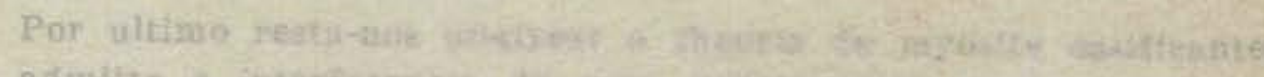

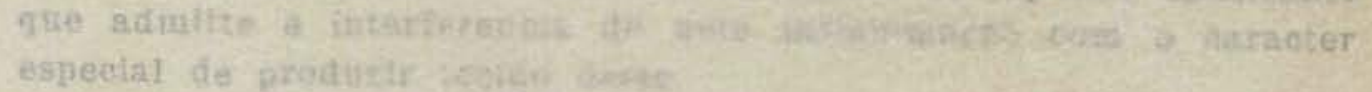

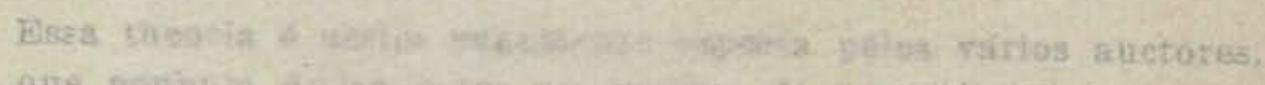

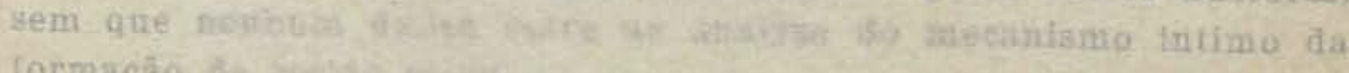

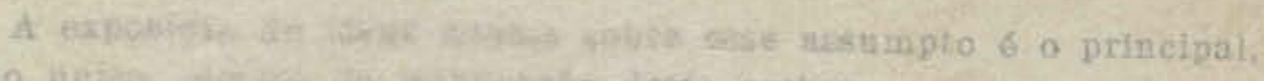

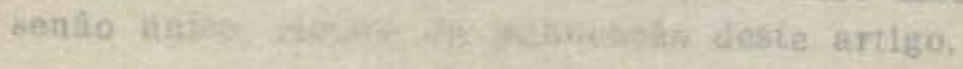

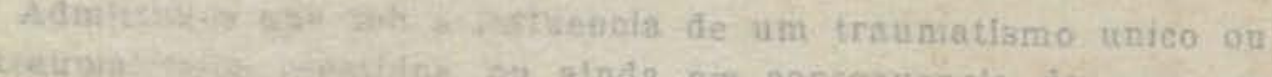

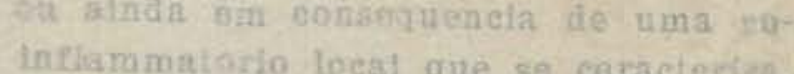
Lamanaçîs; por phenomenos alterativos, faru- 


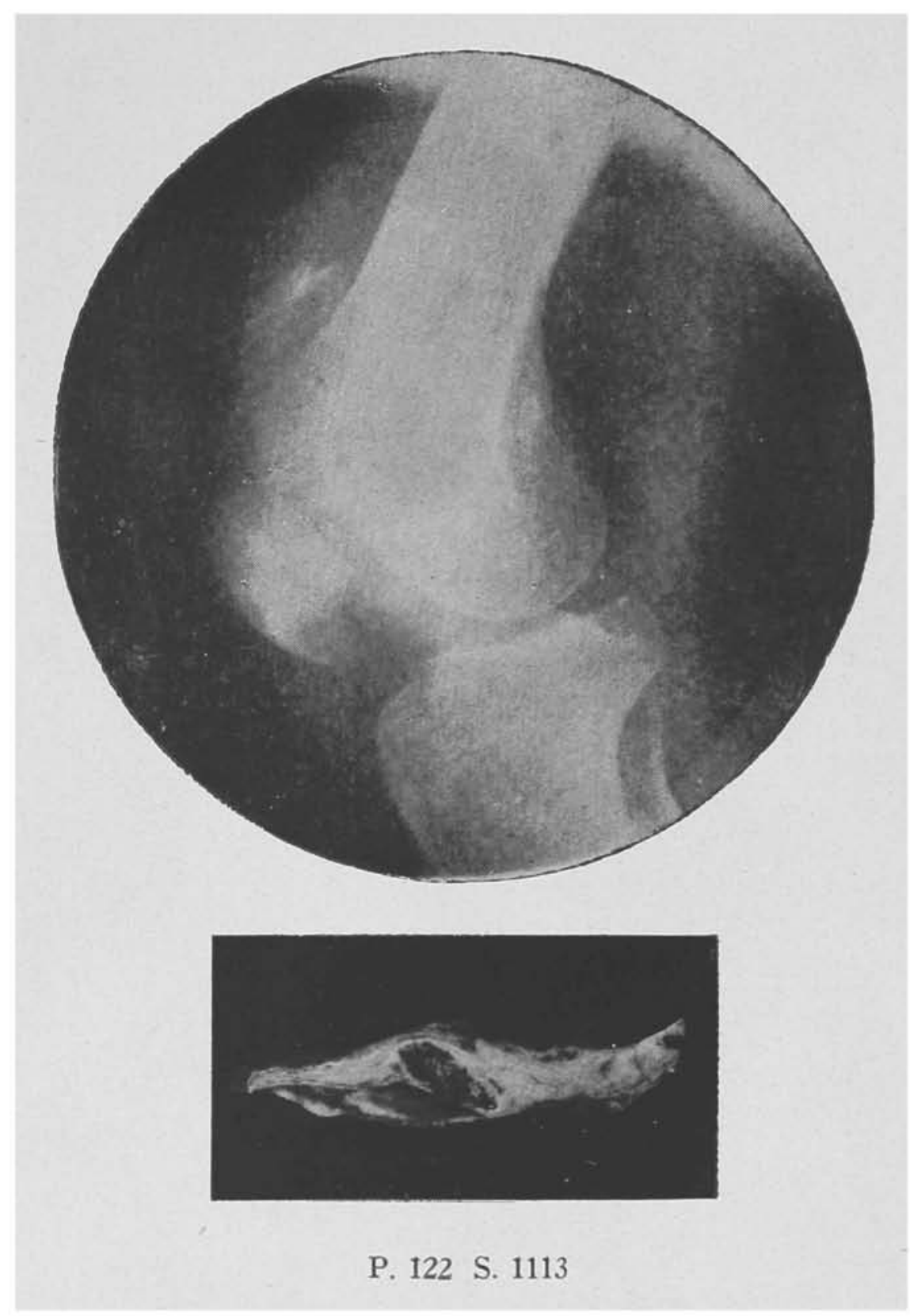



A proliferação regenerativa final é um verdadeiro processo de repazação cicatricial, e assim sendo, encontramos ahi cellulas conjunctivas jovens mais ou menos indifferenciadas, capazes de soffrer a aç̧ão dos factores osteogeneticos que iremos descrever.

W. Roux demonstrou cabalmente a existencia de factores osteogeneticos efficazes e necessarios para a metapiasia oasea. Roux distingue duas ordens de factores: Humoraes e mecanogeneticos̀. Os factores humoraes sâo, no caso em questão, representados por uma constituição especial relacionada provavelmente ao metabolismo do calcio.

Como. factores mecanogeneticc z temos as excitações funccionaes representadas pelas traç̧õe axercidas pela tonicidade propria ao musculo, e, alterativas que se. processam em seguida ás contracções musculares.

Resumindo: Admittimos que em consequencia do processo inf'ammatorio encontram-se nos fócos traumatisados cellulas conjunctivas embryonarias, multipotentes e susceptiveis, portanto, de se influenciar pelos factores normaeõ e communs da osteogenese, os factores de Roux.

As variações do factor humoral explicam a $a_{\tilde{s}}$ tendencias iñ̄i. viduaes maiores ou menores para myosite ossificante, aĩsim como a necessidade dos factores mecanogeneticos esclarece a predilecção dešsas formações para os musculos e tendc̃es.

Assim se explica o que se passou no nosso doente: houve uma ruptura musculo-tendinosa e consequentemente uma phase de prollfєração reparadora cicatricial, que, pela interferencia dos factores cstogeneticos supra descriptos, realisou sua evolução para tecido osseo esponjoso ao envez de restaurar tecido fibroso denso.

Essa noção se coaduna á realidade clinica dos factos e é perfeitamente consentanea com os dados histologicos relatiros á metaplasia ossea.

ANTONIO BERNARDES DE OLIVEIRA.

\section{BIBLIOGRAPHIA}

1) POIICARD - Précis de Histologie Physiologique.

2) LERICHE, R. 'e POLICARD, IN JEANBRAU, NOVE'-JOSSERAND. OMBREDANNE E DESFOSSES. Chirur. repartr. et orthoped. pagina. 150 .

3) FREDET IN JEANBRAU, etc. Chirur. reparatr. et orthoped. pagina. 166.

4) CARLETON - Myositis ossifieans Traumatica. Boston Med. a. Surg. J., $1923,188,390$. 
5) LEWIS, DEAN - Myositis ossifieans. Jour. Amer. Med. Ass., 1923, 80,1281 .

6) BULL, P - Traumatic Ossifyng Myositis. Norsk Magazin for Loegevidenstakaben, Christinia, 1922, 83, 992.

7) PAINTER, C. F. - Etiologic Factors of Myositis Ossifieans. Boston Med. a. Surg. J., 1921, 158, 45.

8) SHERE, OSCAR M. - Myositis ossifieans Traumatiea. Jour. Amer. Med. Ass., 1915, 65, 1012.

9) GRUBER, G. B. - Pathologie Anatomy Circumseribed ossification in a Musele. Nitteilungen a. d. Grenzgebieten der Med. und Chir., Iena. Março $7,817$.

10) OLIVER, J. FAY - Paraosteal Callus: The so-called myositis ossificans traumatica. Western Surgical Assoc. in Jour. Amer. Med. Ass., 1914, 62, 231.

11) GOTO, S. - ossifyng Myositis. Mitteilungen a d. Med. Fak. der Univ., Fukuoka, Japão, 1 N.o 1.

12) OLIVER P. - Myositis Ossifieans following a single trauma. Jour. Amer. Med. Ass., 1914, 63, 1452, com alguma bibliographia.

13) COLEY, W. B. - Myositis ossificans Tramatica. Annals of Surg. Philadelphia, Março, LVII, N.o 3.

14) Stevens, W. E. - Myositis ossifieans Traumatica. Jour. Amer. Med. Ass., 1913, 60, 203.

15) VANCE, ST, OLAIR - A case of Myositis ossifieans Traumatiea. Jour. Amer. Med. Assis., 1.912, 59, 273.

16) NILSON, G. - Ossifseerande myositis i musc. brach. antic. Hygiea, Stuckolm. Março, LXXIV, N.॰ 3 .

17) NAJAPA, C. A. - Myositis ossificans. Indian Med. Gazet., Ca!cutta, Abril, XLVII, N.o 4.

18) EWALD, P - Ossificans Moysitis after trauma. Deutsche Zeitschrift fur Chir., Le!pz!g, Nov. CVII.

19) FABRIS, U. - Origins of Ossifying Myositis. Gazzetta degli Ospedali e delle Cliniche. Mião, Abril 6, XXXII, N.・42.

Attesto que tenho empregado em minha clinica o VIDAN com excellentes resultados.

DR. RUBIÃO MEIRA 